IMA Journal of Applied Mathematics (2000) 64, 39-50

\title{
Microwave Heating of Ceramic Composites
}

\author{
JOHN A. PELESKO \\ Applied Mathematics, California Institute of Technology, Pasadena, California 91125, \\ USA \\ GREGORY A. KRIEGSMANN \\ Department of Mathematical Sciences, Center for Applied Mathematics and Statistics, \\ New Jersey Institute of Technology, University Heights, Newark, New Jersey 07102, USA
}

[Received 25 February 1998 and in revised form 12 April 1999]

\begin{abstract}
The microwave heating of a ceramic composite is modelled and analysed. The composite consists of many small ceramic particles embedded in a ceramic cement. The composite is assumed to be well insulated, and each particle is assumed to be in imperfect thermal contact with the surrounding cement. Based on these two assumptions an asymptotic theory exploiting the small Biot number and small non-dimensional contact conductance is developed. Our asymptotic theory yields a set of nonlinear partial differential equations which govern the temperature in the composite. These are reduced to a set of coupled nonlinear ordinary differential equations in which the surface area of each particle enters as a parameter. Recent experiments with such composites have shown that the steadystate temperature of the composite is strongly dependent upon the radii of the embedded particles. Our model captures this effect. In fact, our analysis shows that the assumption of imperfect thermal contact between the particles and the ceramic cement is essential for this trend to be established.
\end{abstract}

\section{Introduction}

In recent years, microwave processing of ceramic materials has become an area of intense activity (Sutton, 1992). Investigators in the field hope to efficiently produce high-quality materials and products. However, they are faced with numerous difficulties. Some materials of commercial interest, such as alumina, essentially do not absorb microwaves at room temperature, while still others, such as silicon carbide, absorb microwaves readily at any temperature. Some investigators have attempted to enhance the microwave absorption of low-loss materials by embedding many small lossy particles in a low-loss ceramic cement or matrix (Leiser et al., 1997). With this approach, desirable properties of the low-loss cement, such as formability and strength, may be combined with the strong microwave absorption of the lossy material, to manufacture products with a range of useful features. In order to realize this goal, it is necessary to understand the microwave heating of such composites. In this paper, we construct a model of the microwave heating of a class of ceramic composites; this model is then applied to the lossy/low-loss experimental scenario.

We begin by formulating a model of the microwave heating of an arbitrary number of ceramic particles embedded in a ceramic matrix. The model is inherently nonlinear and an exact solution is beyond our reach. We note, however, that experimental situations of current interest (Leiser et al., 1997) suggest several simplifications. That is, noting current 
experimental results suggests a parameter range of interest which allows us to simplify our system. In particular, we develop an asymptotic theory based on the assumption of a small Biot number and a small contact conductance. That is, we assume that the entire sample is well insulated, and that heat transfer from the particles to the matrix is slower than heat transfer across an individual particle. The reduced theory thus obtained consists of a system of nonlinear coupled first-order ordinary differential equations. This reduced system constitutes a leading-order model for the microwave heating of this class of ceramic composites. The analysis of such a system is considerably easier than the analysis of the original model. We carry out such an analysis for a case of experimental interest and show that qualitative agreement with experiment is a feature of this model. In particular, we specialize to the case where the embedded particles possesses identical material properties, disparate electrical properties, and study variations in the steady-state temperature of the system with varying particle radii. Recent experiments (Leiser et al., 1997) have shown that varying the radii of the embedded particles while holding the total mass of the embedded particles fixed causes significant variations in the steady-state temperature of the system. We discuss these experimental results in light of the results of our analysis and offer a possible explanation for the observed behaviour. Finally, we indicate how our model may be used to help design ceramic products with tailor-made microwave heating characteristics.

\section{Formulation of the model}

We consider the microwave heating of a ceramic composite comprising $M$ small particles embedded in a ceramic cement. It is assumed that the particles are uniformly distributed throughout the body, that no two of the particles are in direct contact, and that no two of the particles is exposed to the external environment. That is, each particle is completely surrounded by the matrix. We denote the ceramic region by $\Omega^{\prime}$, and its outer boundary by $\partial_{c}^{\prime}$, while each particle's region is denoted by $\Omega_{i}^{\prime}$, and its outer boundary by $\partial_{i}^{\prime}$. Further, we assume that the electric field is known throughout the sample. This last assumption allows us to focus on the thermal aspect of the problem.

With these assumptions in mind, we first formulate the equation governing temperature evolution in the cement, that is, in the region $\Omega^{\prime}$. The temperature in this region, $T_{c}$, satisfies

$$
\rho_{c} c_{c} \frac{\partial T_{c}}{\partial t^{\prime}}=\kappa_{c} \nabla^{\prime 2} T_{c}+\frac{\left|E_{c}\right|^{2}}{2} \zeta_{c}\left(T_{c}\right) \text { in } \Omega^{\prime},
$$

where $\rho_{c}$ denotes density, $c_{c}$ specific heat, $\kappa_{c}$ thermal conductivity, $\left|E_{c}\right|^{2}$ electric field intensity, $\zeta_{c}$ the effective electrical conductivity, and $\nabla^{\prime 2}$ is the Laplace operator. Similarly, labelling the temperature in the $i$ th particle by $T_{i}$ the temperature in region $\Omega_{i}^{\prime}$ satisfies

$$
\rho_{i} c_{i} \frac{\partial T_{i}}{\partial t^{\prime}}=\kappa_{i} \nabla^{\prime 2} T_{i}+\frac{\left|E_{i}\right|^{2}}{2} \zeta_{i}\left(T_{i}\right) \text { in } \Omega_{i}^{\prime} .
$$

Here, of course, the parameters and variables have the same meaning as above but refer to the material occupying the $i$ th region. 
Next, we formulate the boundary conditions for our problem. Thermal energy is assumed to be convected and radiated at the surface of the sample, hence we assume that

$$
\kappa_{c} \frac{\partial T_{c}}{\partial n^{\prime}}+h\left(T_{c}-T_{A}\right)+s \epsilon\left(T_{c}^{4}-T_{A}^{4}\right)=0 \quad \text { on } \quad \partial_{c}^{\prime} .
$$

Here, $n^{\prime}$ denotes the outward unit normal to the surface, $h$ is a convective heat-transfer coefficient, $s$ is the Stefan-Boltzmann constant, $\epsilon$ is the emissivity, and $T_{A}$ is the ambient temperature of the surrounding environment. Each particle is assumed to be in imperfect thermal contact with the surrounding matrix. Hence on each particle boundary we impose:

$$
\begin{aligned}
\kappa_{i} \frac{\partial T_{i}}{\partial n_{i}^{\prime}} & =\kappa_{c} \frac{\partial T_{c}}{\partial n_{c}^{\prime}} \text { on } \partial_{i}^{\prime}, \\
\kappa_{i} \frac{\partial T_{i}}{\partial n_{i}^{\prime}} & =-k_{i}\left(T_{i}-T_{c}\right) \text { on } \partial_{i}^{\prime},
\end{aligned}
$$

where $k_{i}$ denotes the contact conductance of each boundary region, and the normals are again outward unit normals. Finally, we assume that initially the entire sample is at the ambient temperature and impose

$$
T_{c}\left(\vec{x}^{\prime}, 0\right)=T_{i}\left(\vec{x}^{\prime}, 0\right)=T_{A} .
$$

Next, we choose dimensionless temperature and length scales, scale the electric field with a reference field amplitude, and rewrite the conductivities as their values at the ambient temperature, multiplied by a dimensionless function of the scaled temperature. Further, we scale time with respect to the diffusive time of the cement. This yields the new variables:

$$
\begin{aligned}
& \theta=\frac{T_{c}-T_{A}}{T_{A}}, \quad \phi_{i}=\frac{T_{i}-T_{A}}{T_{A}}, \quad e_{c}=\frac{E_{c}}{E_{0}}, \quad e_{i}=\frac{E_{i}}{E_{0}}, \\
& \zeta_{c}=\sigma_{c} f(\theta), \quad \zeta_{i}=\sigma_{i} g_{i}\left(\phi_{i}\right), \quad t=\frac{\kappa_{c} t^{\prime}}{\rho_{c} c_{c} L^{2}}, \quad \vec{x}=\frac{\vec{x}^{\prime}}{L} .
\end{aligned}
$$

We have assumed that the sample geometry is characterized by a single length scale, $L$. When these dimensionless variables are introduced into our governing equations, we obtain:

$$
\begin{aligned}
& \frac{\partial \theta}{\partial t}=\nabla^{2} \theta+p B f(\theta)\left|e_{c}\right|^{2} \quad \text { in } \Omega, \\
& \mu_{i} \gamma_{i} \frac{\partial \phi_{i}}{\partial t}=\nabla^{2} \phi_{i}+\frac{p B \gamma_{i}}{\delta_{i}} g_{i}\left(\phi_{i}\right)\left|e_{i}\right|^{2} \text { in } \Omega_{i}, \\
& \frac{\partial \theta}{\partial n}+B L(\theta)=0 \quad \text { on } \partial_{c}, \\
& L(\theta)=\theta+R\left[(\theta+1)^{4}-1\right], \\
& \frac{\partial \phi_{i}}{\partial n}=\gamma_{i} \frac{\partial \theta}{\partial n} \quad \text { on } \partial_{i}, \\
& \frac{\partial \phi_{i}}{\partial n}+B_{i}\left(\phi_{i}-\theta\right)=0 \quad \text { on } \partial_{i}, \\
& \theta(\vec{x}, 0)=\phi_{i}(\vec{x}, 0)=0 .
\end{aligned}
$$


Here, the unprimed symbols denoting regions or boundaries refer to scaled versions of these regions; similarly, unprimed normals are dimensionless outward pointing unit normals. Further, the parameters in the above equations are defined as

$$
\begin{aligned}
\mu_{i}=\frac{\rho_{i} c_{i}}{\rho_{c} c_{c}}, \quad \gamma_{i}=\frac{\kappa_{c}}{\kappa_{i}}, \quad p=\frac{E_{0}^{2} \sigma_{c} L}{2 h T_{A}}, \quad \delta_{i}=\frac{\sigma_{c}}{\sigma_{i}}, \\
B=\frac{h L}{\kappa_{c}}, \quad R=\frac{s \epsilon T_{A}^{3}}{h}, \quad B_{i}=\frac{k_{i} L}{\kappa_{i}} .
\end{aligned}
$$

\section{An asymptotic theory}

We note that equations (7) to (13) are nonlinear and that an exact solution is beyond our reach. In this section, we develop an asymptotic theory which allows us to reduce our set of $M+1$ nonlinear partial differential equations to a set of $M+1$ nonlinear ordinary differential equations. As we shall see, the analysis of the reduced system is more tractable. First, let us consider the non-dimensional parameters which arose when we scaled our governing equations. The parameter $\mu_{i}$ may be interpreted as a relative heat capacity as it is simply a ratio of the heat capacity of the $i$ th particle to the heat capacity of the cement. Similarly, $\gamma_{i}$ is a relative thermal conductivity, appearing as a ratio of the thermal conductivity in the cement to the thermal conductivity in the ceramic. The parameter $p$ is a non-dimensional power. In particular it is a ratio of a reference microwave power absorbed by the cement to a reference power lost at the surface due to convection. The parameter $\delta_{i}$ is the ratio of room temperature electrical conductivities in the cement and the $i$ th material, hence it is simply a relative electrical conductivity. The parameter $B$, which is known as the Biot number, measures the relative strengths of convection and conduction. The parameter $R$ is simply the radiative equivalent of the Biot number. Finally, the parameter $B_{i}$ is similar to the Biot number, but measures the relative strengths of conduction across a particle surface to conduction within the particle.

Next, in order to develop an asymptotic theory, we need to order our parameters. Since we would like our model to be general enough to include materials which are not too disparate thermally or electrically, we take the parameters $\mu_{i}, \gamma_{i}$ and $\delta_{i}$ to be $O(1)$. Further, the power $p$, and the radiative Biot number $R$, are also assumed to be $O(1)$. The first assumption implies that power absorbed is the same order of magnitude as power lost, the second allows the effects of radiation and convection to be weighted equally in our model. Next, we assume that the Biot number $B$ is a small parameter. This is equivalent to assuming that the composite is well insulated, which is true in typical experimental situations (Sutton, 1992; Leiser et al., 1997; Kriegsmann, 1992; Pelesko \& Kriegsmann, 1997). Finally, we assume that the parameters $B_{i}$, are small and are the same order as the Biot number. We rescale by setting $B_{i}=\beta_{i} B$, where the $\beta_{i}$ are $O(1)$. Here, we are assuming that it is easier to transport heat through a particle than it is to transport heat through the particle's surface.

With these assumptions, we attempt a regular perturbation expansion, by assuming power-series solutions in the form

$$
\theta \sim \theta_{0}+B \theta_{1}+\cdots, \quad \phi_{i} \sim \phi_{i 0}+B \phi_{i 1}+\cdots .
$$


Inserting these into our governing equations (7) to (13), expanding the nonlinear terms in Taylor series, and equating to zero the coefficients of the powers of $B$, we obtain an infinite set of equations which sequentially should determine the $\theta_{n}$ and $\phi_{i n}$. A quick glance at our governing equations, however, shows that this approach is flawed. In particular it is easy to see that the leading-order solutions will be identically zero, while the $O(B)$ solutions will blow up linearly with time. To rectify this situation, we rescale the time variable with the Biot number, $\tau=B t$, and employ the technique of asymptotic matching. Here, since the leading-order solutions are identically zero, the matching is trivial. That is, the long time behaviour of the leading-order solution on the $t$ time scale gives the initial conditions for the leading-order solution on the $\tau$ time scale, but here the initial conditions do not change, that is, this boundary layer in time is empty. So, we may proceed to analyse our rescaled equations, these are

$$
\begin{aligned}
& B \frac{\partial \theta}{\partial \tau}=\nabla^{2} \theta+p B f(\theta)\left|e_{c}\right|^{2} \quad \text { in } \Omega, \\
& B \mu_{i} \gamma_{i} \frac{\partial \phi_{i}}{\partial \tau}=\nabla^{2} \phi_{i}+\frac{p B \gamma_{i}}{\delta_{i}} g_{i}\left(\phi_{i}\right)\left|e_{i}\right|^{2} \quad \text { in } \Omega_{i}, \\
& \frac{\partial \theta}{\partial n}+B L(\theta)=0 \quad \text { on } \quad \partial_{c}, \\
& L(\theta)=\theta+R\left[(\theta+1)^{4}-1\right], \\
& \frac{\partial \phi_{i}}{\partial n}=\gamma_{i} \frac{\partial \theta}{\partial n} \quad \text { on } \partial_{i}, \\
& \frac{\partial \phi_{i}}{\partial n}+B \beta_{i}\left(\phi_{i}-\theta\right)=0 \quad \text { on } \partial_{i}, \\
& \theta(\vec{x}, 0)=\phi_{i}(\vec{x}, 0)=0 .
\end{aligned}
$$

Technically, we should relabel our dependent variables, as they are now functions of $\tau$; to avoid confusing notation, we shall simply remember that this is true and leave the variable names unchanged. Now, we again attempt an expansion in the form

$$
\theta \sim \theta_{0}+B \theta_{1}+\cdots, \quad \phi_{i} \sim \phi_{i 0}+B \phi_{i 1}+\cdots .
$$

Inserting these into our rescaled equations (14) to (20), expanding nonlinear terms in a Taylor series, and equating to zero coefficients of powers of $B$, we obtain

$$
\begin{aligned}
\nabla^{2} \theta_{0} & =0 \text { in } \Omega, \\
\nabla^{2} \phi_{i 0} & =0 \text { in } \Omega_{i}, \\
\frac{\partial \theta_{0}}{\partial n} & =0 \quad \text { on } \partial_{c}, \\
\frac{\partial \phi_{i 0}}{\partial n} & =\gamma_{i} \frac{\partial \theta_{0}}{\partial n} \quad \text { on } \partial_{i}, \\
\frac{\partial \phi_{i 0}}{\partial n} & =0 \quad \text { on } \partial_{i}, \\
\theta_{0}(\vec{x}, 0) & =\phi_{i 0}(\vec{x}, 0)=0,
\end{aligned}
$$

and

$$
\frac{\partial \theta_{0}}{\partial \tau}=\nabla^{2} \theta_{1}+p f\left(\theta_{0}\right)\left|e_{c}\right|^{2} \quad \text { in } \Omega,
$$




$$
\begin{aligned}
& \mu_{i} \gamma_{i} \frac{\partial_{0} \phi_{i}}{\partial \tau}=\nabla^{2} \phi_{i 1}+\frac{p \gamma_{i}}{\delta_{i}} g_{i}\left(\phi_{i 0}\right)\left|e_{i}\right|^{2} \text { in } \Omega_{i}, \\
& \frac{\partial \theta_{1}}{\partial n}+L\left(\theta_{0}\right)=0 \text { on } \partial_{c}, \\
& \frac{\partial \phi_{i 1}}{\partial n}=\gamma_{i} \frac{\partial \theta_{1}}{\partial n} \quad \text { on } \partial_{i}, \\
& \frac{\partial \phi_{i 1}}{\partial n}+\beta_{i}\left(\phi_{i 0}-\theta_{0}\right)=0 \text { on } \partial_{i}, \\
& \theta_{1}(\vec{x}, 0)=\phi_{i 1}(\vec{x}, 0)=0,
\end{aligned}
$$

where we have only given the first two sets of equations; these are sufficient to determine the leading-order behaviour.

We begin our analysis by noting that equations (21) to (26) immediately imply that $\theta_{0}=\theta_{0}(\tau)$ and $\phi_{i 0}=\phi_{i 0}(\tau)$, that is, our leading-order solutions are functions of time only. Next, we derive a system of ordinary differential equations which determine the evolution of $\theta_{0}$ and $\phi_{i 0}$. We integrate equation (27) over the volume $\Omega$ to obtain

$$
V_{c} \frac{\mathrm{d} \theta_{0}}{\mathrm{~d} \tau}=\int_{\Omega} \nabla^{2} \theta_{1}+p f\left(\theta_{0}\right)\left\|e_{c}\right\|^{2}
$$

where

$$
\left\|e_{c}\right\|^{2}=\int_{\Omega}\left|e_{c}\right|^{2}
$$

and $V_{c}$ denotes the volume of the cement. Now, we use the divergence theorem to rewrite the integral as a surface integral and apply the boundary conditions (29) to (31) to obtain

$$
\int_{\Omega} \nabla^{2} \theta_{1}=-S_{c} L\left(\theta_{0}\right)+\sum_{i} \frac{\beta_{i} S_{i}}{\gamma_{i}}\left(\phi_{i 0}-\theta_{0}\right),
$$

where here, $S_{c}$ is the surface area of the cement, $S_{i}$ is the surface area of the $i$ th particle, and the summation is over all $M$ particles. Inserting equation (35) into equation (33) yields

$$
V_{c} \frac{\mathrm{d} \theta_{0}}{\mathrm{~d} \tau}=p f\left(\theta_{0}\right)\left\|e_{c}\right\|^{2}-S_{c} L\left(\theta_{0}\right)+\sum_{i} \frac{\beta_{i} S_{i}}{\gamma_{i}}\left(\phi_{i 0}-\theta_{0}\right) .
$$

Next, integrating equation (28) over $\Omega_{i}$, using the divergence theorem in the same manner as above, and using the boundary conditions (29) to (31), we obtain

$$
\mu_{i} \gamma_{i} V_{i} \frac{\mathrm{d} \phi_{i 0}}{\mathrm{~d} \tau}=\frac{p \gamma_{i}}{\delta_{i}} g_{i}\left(\phi_{i 0}\right)\left\|e_{i}\right\|^{2}-\beta_{i} S_{i}\left(\phi_{i 0}-\theta_{0}\right)
$$

where here $V_{i}$ denotes the volume of the $i$ th particle. Equations (36) and (37) together with the appropriate initial conditions constitute a set of $M+1$ nonlinear coupled ordinary differential equations for the leading-order behaviour of the temperature in the cement and in each particle. 


\section{Analysis}

In this section, we apply our simplified model, embodied in equations (36) and (37), to an experimental situation of current interest. In particular, we specialize to the case of a single species of embedded particles. That is, we shall assume that the $M$ embedded particles are identical with regards to size, material properties etc. Further, we assume that the electric field is known and piecewise constant throughout the sample. The field is assumed to have the constant amplitude $E_{c}$ in the cement and $E_{i}$ in the particles. Note that this implies that field intensities may be assumed scaled to one. This assumption is valid provided the sample size is smaller than an electromagnetic wavelength. At typical microwave frequencies, a wavelength is on order of ten centimeters. So, our results are valid for samples characterized by a length scale on order of one to two centimeters. We further note, that for dilute mixtures, changing the particle radius will not effect the field in the particles and that the effect on the field in the cement will be negligible for the thermal problem. To clarify, we are assuming perfect electrical contact and focusing our attention on the effects of imperfect thermal contact.

With these two assumptions, our set of $M+1$ equations may be reduced to two equations. First, equation (36) simplifies to

$$
V_{c} \frac{\mathrm{d} \theta_{0}}{\mathrm{~d} \tau}=p V_{c} f\left(\theta_{0}\right)-S_{c} L\left(\theta_{0}\right)+\frac{\beta S_{p}}{\gamma}\left(\phi_{0}-\theta_{0}\right),
$$

where $S_{p}$ denotes the total surface area of all the embedded particles, subscripts on $\beta$ and $\gamma$ have been dropped to indicate that they refer to the properties of any given particle, and $\phi_{i 0}$ has been replaced by $\phi_{0}$ to indicate that we need only track the temperature of one particle. To obtain an equation for $\phi_{0}$ we may select any one of the $M$ equations, (37). Selecting any one of these and multiplying by $M$ for convenience yields

$$
\mu \gamma V_{p} \frac{\mathrm{d} \phi_{0}}{\mathrm{~d} \tau}=\frac{p V_{p} \gamma}{\delta} g\left(\phi_{0}\right)-\beta S_{p}\left(\phi_{0}-\theta_{0}\right),
$$

where here, $V_{p}$ denotes the total volume of all the embedded particles and again subscripts have been dropped to indicate that the material properties are identical for all particles.

Now, thus far we have reduced our system of governing equations by specializing to the experimentally interesting situation where a single species of particles is present. We further specialize to the situation where the cement may be considered low-loss, while the embedded particles are assumed lossy. For example, the cement may be alumina, while the embedded particles may be silicon carbide (Leiser et al., 1997). Examination of conductivity data for such materials (Westphal, 1963) dictates the form of the heretofore unspecified functions, $f\left(\theta_{0}\right)$ and $g\left(\phi_{0}\right)$. In particular, the conductivity of the particles may be assumed to be a weak function of temperature, and hence we may choose $g\left(\phi_{0}\right)=1$. In contrast, the conductivity of the cement must be assumed to be a strong function of temperature, typically exponential, and we choose $f\left(\theta_{0}\right)=\exp \left(a \theta_{0}\right)$.

Throughout the remainder of this section we will study

$$
\begin{aligned}
V_{c} \frac{\mathrm{d} \theta_{0}}{\mathrm{~d} \tau} & =p V_{c} f\left(\theta_{0}\right)-S_{c} L\left(\theta_{0}\right)+\frac{\beta S_{p}}{\gamma}\left(\phi_{0}-\theta_{0}\right), \\
\mu \gamma V_{p} \frac{\mathrm{d} \phi_{0}}{\mathrm{~d} \tau} & =\frac{p V_{p} \gamma}{\delta}-\beta S_{p}\left(\phi_{0}-\theta_{0}\right) .
\end{aligned}
$$


We begin our analysis by seeking steady-state solutions. Such solutions must satisfy

$$
\begin{gathered}
p V_{c} f\left(\theta_{0}^{*}\right)-S_{c} L\left(\theta_{0}^{*}\right)+\frac{\beta S_{p}}{\gamma}\left(\phi_{0}^{*}-\theta_{0}^{*}\right)=0, \\
\frac{p V_{p} \gamma}{\delta}-\beta S_{p}\left(\phi_{0}^{*}-\theta_{0}^{*}\right)=0 .
\end{gathered}
$$

We may solve the first of these equations for $\phi_{0}^{*}$ and eliminate in favour of $\theta_{0}^{*}$ in the second to obtain

$$
p=\frac{S_{c} L\left(\theta_{0}^{*}\right)}{V_{c} f\left(\theta_{0}^{*}\right)+V_{p} / \delta} .
$$

This is reminiscent of a typical power-response curve for a homogenous material (Kriegsmann, 1992). In general, we cannot explicitly solve (42) for $\theta_{0}^{*}$ as a function of $p$. However, this analysis can be done graphically as shown in Fig. 1, where $p$ is plotted as a function of $\theta_{0}^{*}$ and the axes are reversed. Note that this power response curve has only two branches, in contrast to the typical S-shaped power-response curves usually observed. Recall that we have assumed that the electric field is a constant, in (Kriegsmann, 1992) it was shown that for homogeneous materials, including temperature variations of the electric field restores the upper branch of the S-shaped curve. In addition, in (Pelesko \& Kriegsmann, 1997) it was shown that a similar phenomenon occurs in the heating of ceramic laminates composed of two electrically disparate materials. We do not do this here; rather we simply conjecture that our upper branch will be restored if the full electric field is computed as a function of temperature.

We also observe here that $\theta_{0}^{*}$ does not depend upon $\beta$, that is, the steady-state cement temperature is independent of the imperfect thermal contact between the particles and the cement. This follows from the fact that the cement is essentially insulated from its surroundings (see equation (16)) and the particles (see equations (18), (19)) in the small Biot number limit. Higher-order corrections to the cement temperature will depend upon $\beta$.

We may study the stability of our steady-state solutions by linearizing our governing equations near each critical point. We carry this out by first inserting the ansatz

$$
\theta_{0}=\theta_{0}^{*}+A \mathrm{e}^{\lambda \tau}, \quad \phi_{0}=\phi_{0}^{*}+B \mathrm{e}^{\lambda \tau}
$$

into our governing equations and expanding nonlinear terms in a Taylor series. We find that $\lambda$ satisfies

$\lambda^{2}+\left(\frac{\beta S_{p}}{\mu \gamma V_{p}}+\frac{\beta S_{p}}{\gamma V_{c}}+p^{\prime}\left(\theta_{0}^{*}\right)\left(f\left(\theta_{0}^{*}\right)+\frac{V_{p}}{\delta V_{c}}\right)\right) \lambda+\frac{\beta S_{p}}{\mu \gamma V_{p}}\left(f\left(\theta_{0}^{*}\right)+\frac{V_{p}}{\delta V_{c}}\right) p^{\prime}\left(\theta_{0}^{*}\right)=0$,

where the prime on $p$ denotes differentiation with respect to $\theta_{0}^{*}$. Now, by noting that $p^{\prime}$ is positive on the lower branch and negative on the upper branch, we immediately see that the lower branch is stable, while the upper branch is unstable.

Next, having found and determined the stability of steady-state solutions, we would like to see if the results of this analysis agree with experiment. In particular, we would like 


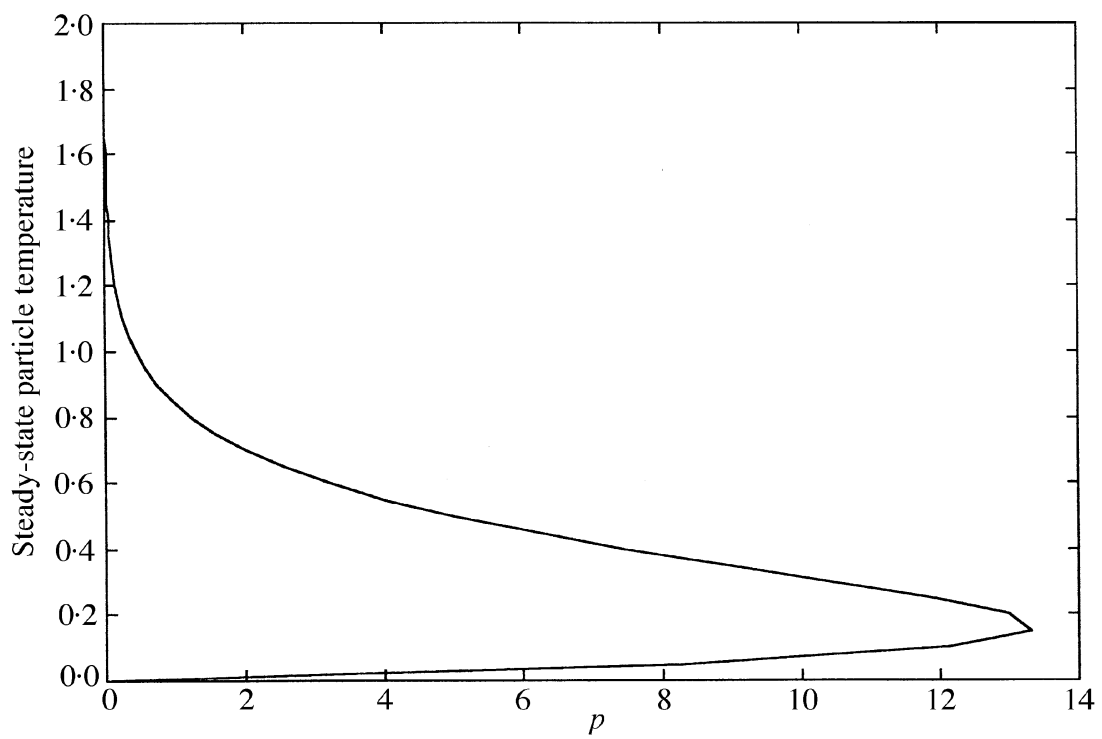

FIG. 1. A typical power-response curve.

to determine whether or not the steady-state temperature of the sample varies with varying particle radii for a fixed total mass of embedded particles. However, we are immediately faced with a dilemma. The steady-state temperature of the particles is in general different than that of the cement! That is, while the steady-state temperature of the cement, that is, of the $\theta_{0}^{*}$ are given by (42), the steady-state temperature of each particle is given by

$$
\phi_{0}^{*}=\theta_{0}^{*}+\frac{p V_{P} \gamma}{\delta \beta S_{p}} .
$$

This leads us to inquire as to what temperature is actually measured experimentally. Since the diameter of a typical thermocouple inserted into the sample is much larger than a particle diameter, it seems reasonable to assume that the experimentally measured temperature is some weighted average of particle and cement temperatures. If we multiply equation (40) by $\gamma$ and add it to equation (41), we obtain

$$
\frac{\mathrm{d}}{\mathrm{d} \tau}\left(V_{c} \theta_{0}+\mu V_{p} \phi_{0}\right)=p V_{c} f\left(\theta_{0}\right)+\frac{p V_{p}}{\delta}-S_{c} L\left(\theta_{0}\right)
$$

The right-hand side is simply the net power into the sample and this suggests that we define

$$
\psi=\frac{V_{c} \theta_{0}+\mu V_{p} \phi_{0}}{V_{c}+\mu V_{p}}
$$

as the mean sample temperature. We note that this is a heat capacity weighted volume average. 


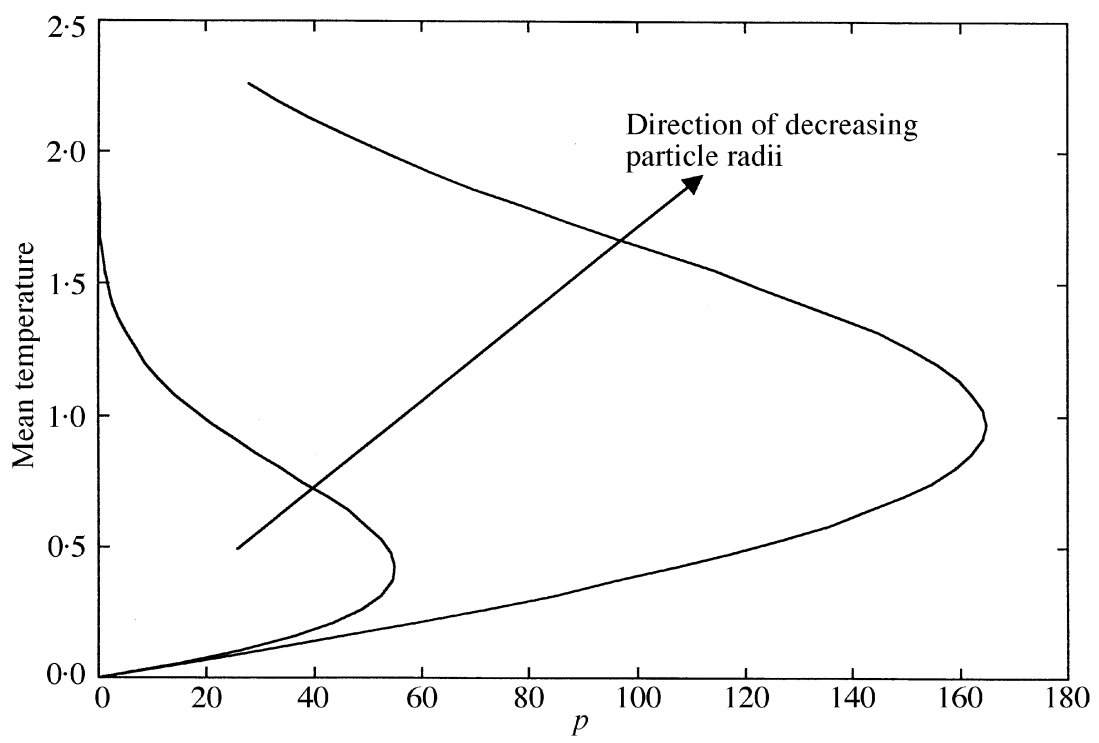

FIG. 2. Variation in power response with particle radii.

Denoting the steady-state value of the mean sample temperature by $\psi^{*}$, it is found from (46) and (48) to be equal to

$$
\psi^{*}=\theta_{0}^{*}+\left(\frac{\mu V_{p}}{V_{c}+\mu V_{p}}\right)\left(\frac{p V_{p} \gamma}{\delta \beta S_{p}}\right)
$$

Now, we may make some qualitative comparisons with experiment. As mentioned earlier, it has been observed that the steady-state temperature of the sample, $\psi^{*}$ decreases with decreasing particle radii (Leiser et al., 1997). It is important to note that in these experiments the total mass of the embedded particles is held constant. This implies that in our reduced system only the parameter $S_{p}$ varies with particle radii. Assuming that the particles are spheres, then we have explicitly $S_{p}=3 V_{p} / a$, where $a$ is the radius of a particle. The expression for $\psi^{*}$ now becomes

$$
\psi^{*}=\theta_{0}^{*}+\left(\frac{\mu V_{p}}{V_{c}+\mu V_{p}}\right)\left(\frac{p \gamma}{3 \delta \beta}\right) a
$$

which shows that the steady-state mean temperature increases linearly with particle radius for a fixed volume of particles. This is shown in Fig. 2. Notice that the height of the lower branch decreases with decreasing particle radii. That is, stable steady-state temperatures decrease with decreasing particle radii, in agreement with experimental observations. Finally, in Fig. 3, we plot numerical solutions for $\psi$ as a function of time for various values of $a$. We note that the approach to the steady state is monotonic, and that the curve obtained qualitatively resembles experimental results (Leiser et al., 1997). 


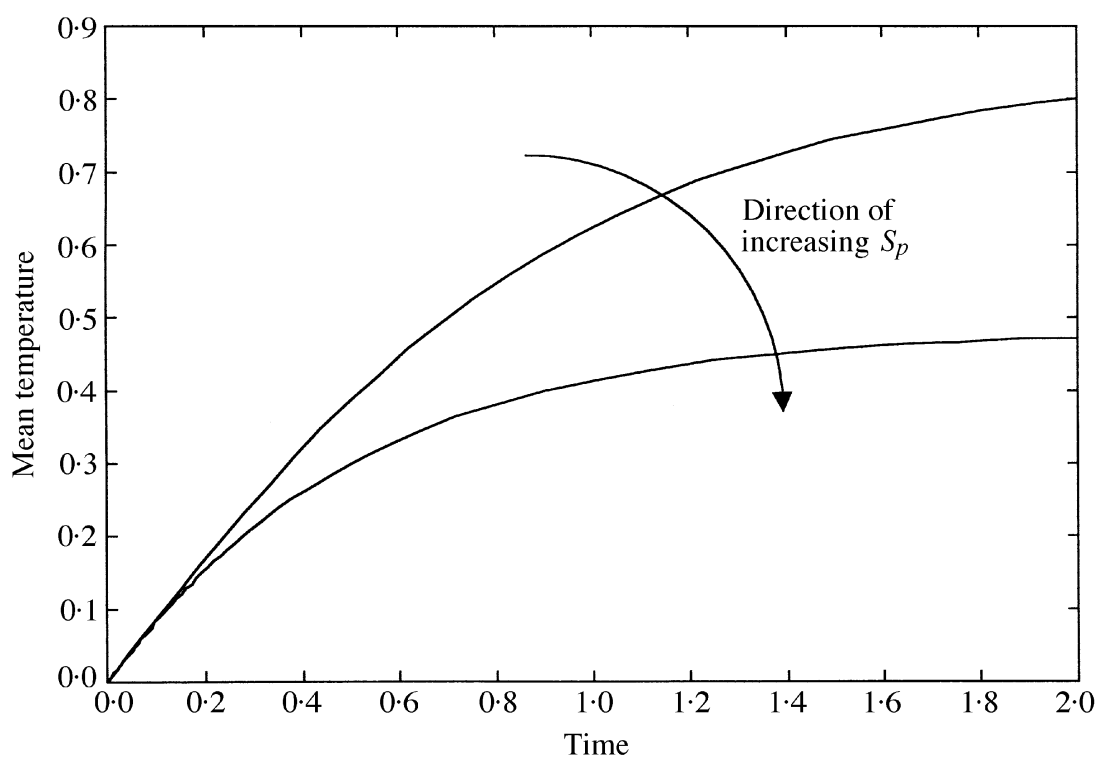

FIG. 3. Variation of mean temperature with particle radii.

\section{Discussion}

We began by constructing a model of the microwave heating of a ceramic composite consisting of many ceramic particles embedded within a ceramic cement. We noted that this model is nonlinear and that in general analytical solutions are not available. Next, we employed asymptotic methods to derive a reduced or simplified model. Two key assumptions were made. First, we assumed that the Biot number was small. That this is the regime of interest is clear; in most experiments the samples are well insulated. We also assumed that the non-dimensional contact conductance, $B_{i}$, was a small parameter. That this should be true is not as obvious. Further, there are very few measured values of contact conductance available. We note, however, that this assumption is strongly suggested by experimental evidence combined with a mathematical observation. As discussed previously, steady-state temperatures are observed to vary strongly with particle radii, even as the total mass of the embedded particles is held constant. Discounting the electric field, which should not vary significantly under these circumstances, the only parameter varied in these experiments is the total surface area of the embedded particles, $S_{p}$. So, in any correct model, the leading-order approximation to the steady-state temperature should vary with $S_{p}$. In the limit of perfect thermal contact, these variations disappear from the solution. This suggests that $B_{i}$ is not large. Further, if $B_{i}$ is taken to be $O(1)$, then to leading-order, the temperature is constant throughout the sample and again variations with $S_{p}$ disappear from the leading-order solution. That suggests that $B_{i}$ is indeed a small parameter.

Next, we applied our simplified model to the experimental situation which suggested the parameter range of interest, and which suggested further simplifications. In particular, we considered the case of lossy particles embedded in a low-loss cement. This assumption 
specified the form of the electrical conductivity functions and allowed us to analyse the steady states of the system. We noted that there was a need to define a mean temperature and we hypothesized that this was the temperature measured in the experiment. We saw that observed experimental results could be explained as a geometric effect; variations in the total surface area of the embedded particles correspond to the observed behaviour for varying particle radii. We also noted that a multi-valued power-response curve for the mean steady-state temperature exists. As in the case of homogeneous materials, it is interesting to consider this curve in the context of thermal runaway. In homogeneous materials, sudden uncontrolled heating of the material occurs once the power is turned past some critical value. On any of our power-response curves, this would correspond to turning the power past the 'nose' of the curve. Here, the same phenomenon occurs. However, we see that by varying particle properties, the location of this 'nose' may be varied. In particular, by simply increasing $S_{p}$, that is, by using smaller particles, the 'nose' moves up and to the right. This allows stable heating of the composite at higher temperatures.

Finally, we note that we have barely scratched the surface in analysing our simplified model of the microwave heating of ceramic composites. Here, we have studied solutions of our model equations in the context of currently known experimental results. If in these simple cases, the quantitative validity of this model could be confirmed, that is, if extensive enough experimental data become available so that such an analysis could be carried out, we would suggest that this model might be useful in designing composites with tailor-made microwave heating properties.

\section{Acknowledgments}

This work was sponsored by the Department of Energy under grant DE-FG02-94ER25196, the National Science Foundation under grant DMS-9407196, and the Air Force Office of Scientific Research, Air Force Materiel Command, USAF, under grant F49620-96-1-0039.

The authors thank both reviewers for their careful reading of the paper and Professor Oscar Bruno for several useful discussions.

\section{REFERENCES}

SutTON, W. H. 1992 Key issues in microwave process technology. In Microwave Processing of Materials III. Pittsburgh, PA: MRS Symposium Proceedings, 3-20.

Leiser, K. S., Di Fiore, R. R., CozzI, A. D., \& Clark, D. E. 1997 Microwave heating rates of silicon carbide/alumina cement susceptors. Ceramic Engineering and Science Proceedings, 18, 551-555, American Ceramic Society.

Kriegsmann, G. A. 1992 Thermal runaway in microwave heated ceramics: A one-dimensional model. Journal of Applied Physics 71, 1960-1966.

WestphaL, W. B. 1963 Dielectric constant and loss measurements on high-temperature materials, Laboratory for Insulation Research Technical Report, MIT, October.

Pelesko, J. A. \& KRIEgsmann, G. A. 1997 Microwave heating of ceramic laminates. Journal of Engineering Mathematics 32, 1-18. 